\title{
Discrimination of Malignant and Benign Breast Masses Using Computer-Aided Diagnosis from Dynamic Contrast-Enhanced Magnetic Resonance Imaging
}

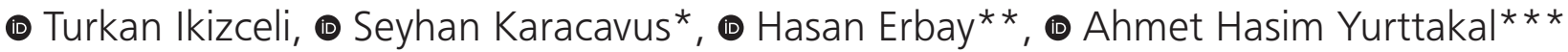 \\ University of Health Sciences Turkey, Istanbul Haseki Training and Research Hospital, Clinic of Radiology, Istanbul, Turkey \\ *University of Health Sciences Faculty of Medicine, Department of Nuclear Medicine, Kayseri, Turkey \\ **Turkish Aeronautical Association University Faculty of Engineering, Department of Computer Engineering, Ankara, Turkey \\ ${ }^{* * *}$ Afyon Kocatepe University Faculty of Engineering, Department of Computer Engineering, Afyon, Turkey
}

\section{Abstract}

\begin{abstract}
Aim: To reduce operator dependency and achieve greater accuracy, the computer-aided diagnosis (CAD) systems are becoming a useful tool for detecting noninvasively and determining tissue characterization in medical images. We aimed to suggest a CAD system in discriminating between benign and malignant breast masses.
\end{abstract}

Methods: The dataset was composed of 105 randomly breast magnetic resonance imaging (MRI) including biopsy-proven breast lesions (53 malignant, 52 benign). The expectation-maximization (EM) algorithm was used for image segmentation. 2D-discrete wavelet transform was applied to each region of interests (ROIs). After that, intensity-based statistical and texture matrix-based features were extracted from each of the 105 ROls. Random Forest algorithm was used for feature selection. The final set of features, by random selection base, splatted into two sets as $80 \%$ training set ( $84 \mathrm{MRI}$ ) and $20 \%$ test set $(21 \mathrm{MRI})$. Three classification algorithms are such that decision tree (DT, C4.5), naive bayes (NB), and linear discriminant analysis (LDA) were used. The accuracy rates of algorithms were compared.

Results: C4.5 algorithm classified 20 patients correctly with a success rate of $95.24 \%$. Only one patient was misclassified. The NB classified 19 patients correctly with a success rate of $90.48 \%$. The LDA Algorithm classified 18 patients correctly with a success rate of $85.71 \%$.

Conclusion: The CAD equipped with the EM segmentation and C4.5 DT classification was successfully distinguished as benign and malignant breast tumor on MRI.

Keywords: Breast lesions, breast cancer, magnetic resonance imaging, computer-aided diagnosis, segmentation

\section{Introduction}

The most crucial role in breast cancer treatment is early detection. Mammography is one of the most valuable tools for detecting breast cancer early before physical symptoms appear $(1,2)$. However, mammographic features of earlystage breast cancer cannot be clear and very specific (3). At present, magnetic resonance imaging (MRI) provides superior soft-tissue imaging capability and is considered the most accurate technique for detecting breast cancer (4). However, the interpretation of MRI images is both times consuming and requires an experienced radiologist. At the same time, using breast MRI for screening breast cancer is gradually increasing as well as the MRI is now being used as a screening modality in the high-risk women population (5). Also, the use of MRI is directly or indirectly associated with higher costs. One reason for the higher price is that the long acquire time of the standard protocol currently used and the length of the reading time. A typical MRI study includes thousands of images (6).

In recent years, machine learning and computeraided techniques are increasingly gaining performance in radiology. To reduce operator dependency and achieve

Address for Correspondence: University of Health Sciences Turkey, Istanbul Haseki Training and Research Hospital, Department of Radiology, Istanbul, Turkey Phone: +90 2125294400 E-mail: ikizceli@yahoo.com ORCID: orcid.org/0000-0002-5683-0391 Received: 16.12.2020 Accepted: 18.02.2021 
greater accuracy, the computer-aided diagnosis (CAD) system is becoming a useful tool for detecting noninvasively and determining tissue characterization in medical images, which leads to an image-based diagnosis. In this way, CAD becomes a necessary tool to help radiologists not only detect tumors but also interpret lesions and avoid unnecessary breast biopsies $(7,8)$.

The CAD, which is seen as the second reader in the clinic, needs to be developed further. The CAD has four main modules: pre-processing, segmentation, feature extraction and selection, and classification (9). Image segmentation is the most important module. There are numerous mass segmentation studies in the literature, but studies on breast segmentation are limited, particularly on breast MRI images (10). One of the segmentation methods, the expectation-maximization (EM) method, has been used for breast density estimation before (11). The most used classifier in literature is k-nearest neighbors (KNN) and supports vector machines (SVM). However, studies with decision trees (DT), linear discriminant analysis (LDA), and naive bayes (NB) as a classification algorithm with MRI are less seen (12).

In this study, a recent user-independent time-saving CAD to diagnose breast cancer using MRI images was presented. Here, we aimed to find the best method for the discrimination of benign and malignant breast masses by comparing the classification methods (LDA, NB, DT) and using EM for segmentation, which has been an unused segmentation on breast MRI images until now.

\section{Methods}

\section{Study Design}

The present study was approved by the Kayseri City Hospital of Medicine Ethics Committee (number; 2020/73) and conducted between form January 2018 to January 2019 in accordance with the Helsinki Declaration. Consent form was filled out by all participants.

In the study, we used a dataset of 105 breast MRIs randomly selected from our breast department's archive, which includes both benign and malignant lesions. Patients with multiple lesions in the breast MRI, infection, granulomatous mastitis, and patients that their MRI was not enough enchantment for image analysis were excluded from the study. All lesions were verified histopathologically by sonographic guidance using a 14-gauge automatic core-needle biopsy.

All breast MR imaging studies were performed using a 1.5-Tesla MRI (Achieva, Philips, The Netherlands) unit with the patients in a prone position using dedicated eightchannel breast coils. Breast MRI images were obtained as standard with fat suppression FATSET in axial plane using T1 turbo spin-echo sequence. T2W STIR, Contrast examinations (DCE-MRI) were obtained with T1 FATSET and THRIVE sequences in the axial plane. Subtraction images were obtained automatically by the device.

\section{Image Analysis}

Pre-processing: A single slice from each MRI image in which the tumor was the most clearly seen in the contrastenhanced sequence was selected as the representing image for the corresponding MRI image. Then, the noise of the image was reduced using the median filter, Gauss filter and the "top hat and bottom hat" methods. In this way, the picture became brighter and the contrast between the two colors was increased.

Segmentation: The EM algorithm (13) was used for segmentation, which is one of the most critical steps of image analysis. First, an elliptic curve placed on the tumorous region on the image manually. The entire lesion was included in the curve. The segmentation process was applied to the area within the curve, each corresponded to a single region of interest (ROI). At the end of the segmentation process, a total of $105 \mathrm{ROI}$ was obtained from a total of $105 \mathrm{MRI}$ images, and they formed the dataset. Each ROI has only two colors, white and black. The tumor tissue was determined as white and non-tumor tissue as black. Moreover, the tumor boundaries were clarified correctly.

2D Discrete Wavelet Transforms: Each of the segmented images were applied to a 2D-discrete wavelet transform before the texture analysis. Then, these filter results were again passed through low and high pass filters using the same filter coefficients and repeated sample reduction was performed.

Feature Extraction: The intensity-based statistical (histogram) and texture matrix-based features [Gray level co-occurrence matrix, (GLCM)] families were used.

Feature Selection: Random forest (RF) was used for feature selection and the Gini index was used as the feature selection property.

Classification Algorithms: The dataset was randomly splatted into two distinct data sets, such as $80 \%$ training set (84 patient's images) and 20\% test set (21 patient's images) for training and testing purposes (Table 1).

Three different classification algorithms (DT, NB and LDA) were employed for classification. C4.5 DT was used. The DT generated by C4.5 can be used for classification, and therefore C4.5 is often referred to as a statistical classifier and a benchmark DT program.

\begin{tabular}{|l|l|l|}
\hline \multicolumn{3}{|c|}{ Table 1. Patient distribution in the training and testing data sets } \\
\hline & $\begin{array}{l}\text { Malign } \\
(\mathbf{n}=53)\end{array}$ & $\begin{array}{l}\text { Benign } \\
(\mathbf{n = 5 2 )}\end{array}$ \\
\hline Training group, $\mathrm{n}(\%)$ & $44(83 \%)$ & $40(77 \%)$ \\
\hline Testing group, $\mathrm{n}(\%)$ & $9(17 \%)$ & $12(23 \%)$ \\
\hline
\end{tabular}




\section{Statistical Analysis}

The correlation between the two groups of datasets was evaluated by the Pearson correlation matrix.

\section{Results}

The dataset was composed of 105 breast MRI (53 malignant, 52 benign). The mean age was $41.40 \pm 10.93$ years (age range, 18-66 years) for benign lesions, $48.40 \pm 11.32$ years (age range, $28-81$ years) for malignant lesions. The lesion's sizes were between $16.57 \pm 4.93 \mathrm{~mm}$ $(10-30 \mathrm{~mm})$ for benign lesions, $31.03 \pm 18.00(7-85 \mathrm{~mm})$ for malignant lesions. The demographic features of the patients were summarized in Table 2.

\section{Image Analysis Results}

The original image in Figure 1a was preprocessed. Then, as the ROI, an elliptic region in Figure 1b, which contained the entire tumorous region, was selected. Later, the ROI was applied to additional preprocessing procedures to obtain the image in Figure 1c. The final image (Figure 1d) was segmented.

The segmented images were applied to the 2D-discrete wavelet transform. After that, four colored images $(C D, C H$, $\mathrm{CV}$, and $\mathrm{CA}$ ) were created as a result of texture analysis.

\begin{tabular}{|c|c|c|}
\hline & $\begin{array}{l}\text { Malign } \\
(n=53)\end{array}$ & $\begin{array}{l}\text { Benign } \\
(n=52)\end{array}$ \\
\hline Age (mean \pm SD) & $48.40 \pm 11.32$ & $41.40 \pm 10.93$ \\
\hline Size $(\mathrm{mm})($ mean \pm SD) & $31.03 \pm 18.00$ & $16.57 \pm 4.93$ \\
\hline \multicolumn{3}{|l|}{ Malign n (\%) } \\
\hline Invasive ductal carcinoma & $35(66 \%)$ & - \\
\hline Lobular carcinoma & $6(11.3 \%)$ & - \\
\hline Ductal carcinoma in situ & $12(22.6 \%)$ & - \\
\hline \multicolumn{3}{|l|}{ Benign n (\%) } \\
\hline Fibroadenomas & - & $42(80.7 \%)$ \\
\hline Sclerosing adenosis & - & $7(13.4 \%)$ \\
\hline Intramamarian lymph node & - & $2(3.8 \%)$ \\
\hline Lactation adenoma & - & $1(1.9 \%)$ \\
\hline \multicolumn{3}{|l|}{ SD: Standard deviation } \\
\hline
\end{tabular}

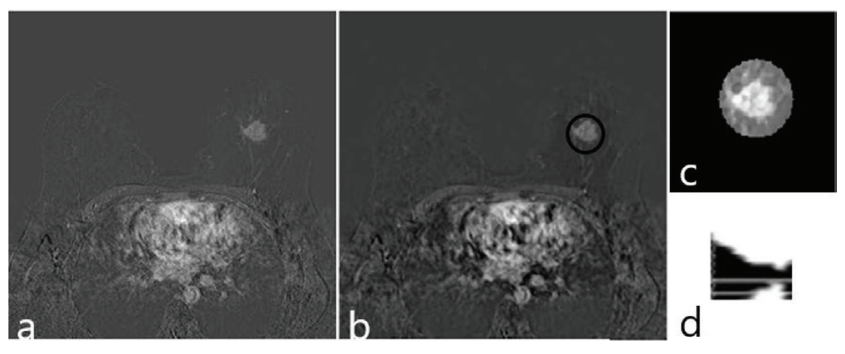

Figure 1. (a) Original image, (b) Preprocessed image and selected ROI, (c) Preprocessed ROI, (d) Segmented ROI ROI: Region of interest
Nine different features were extracted from the four colored images. Figure 2 shows feature importances. The plot has shown that the four features we used were more informative and the rest were less informative.

\section{Classification Performance}

Table 3 shows the output as the confusion matrix. According to the table, C4.5 DT ranked 20 patients properly (95.24\% success rate). Only one patient is misclassified. NB properly categorized 19 patients $(90.48 \%$ success rate). LDA accurately categorized 18 patients (85.71\% success rate). The C4.5DT algorithm is more accurate than the others.

\section{Discussion}

Advances in both imaging and computers have synergistically led to a rapid rise in the potential use of image processing techniques (14). We know that images are more than pictures; they are data. Radiologists have started to show great interest in the clinical use of this data. Images are converted to high-dimensional data and used in decision support of precision medicine (15). The most important limitations are the lack of efficient and standardized systems of feature extraction and

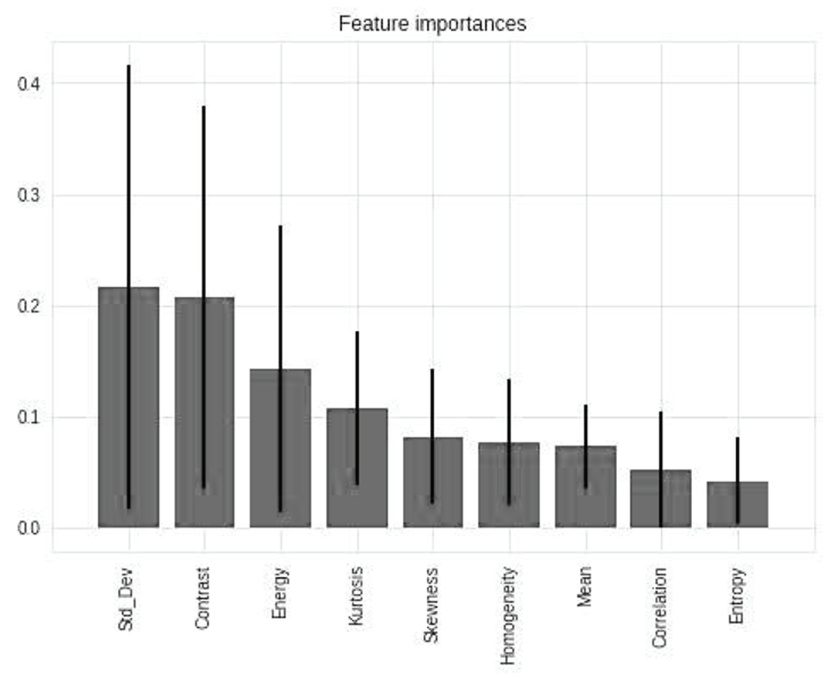

Figure 2. Feature importances

\begin{tabular}{|l|l|l|l|l|l|l|}
\hline Table 3. Classification report \\
\hline & C4.5 & \multicolumn{3}{l|}{ NB } & \multicolumn{2}{l|}{ LDA } \\
\hline & True & False & True & False & True & False \\
\hline True & 12 & 0 & 11 & 1 & 10 & 2 \\
\hline False & 1 & 8 & 1 & 8 & 1 & 8 \\
\hline Mean AUC & 0.80 & 0.79 & 0.60 & \\
\hline Precision & 1.0 & 0.88 & 0.80 \\
\hline Accuracy (\%) & 95.2 & 90.4 & 85.7 \\
\hline AUC: Area under curve, NB: Naive bayes, LDA: Linear discriminant analysis \\
\hline
\end{tabular}


data exploring $(16,17)$. Nevertheless, in comparison with mammography and ultrasound, relatively few CAD systems have been developed for breast MRI in the literature $(16,18)$.

The CAD consists of two major steps: analysis and diagnosis. The analysis step comprises a series of procedures such as preprocessing, segmentation and feature extraction. The diagnosis stage includes the classification procedure (19). Along with the segmentation, the classification module is regarded as the heart of the CAD (20).

Accurate segmentation of the $\mathrm{ROI}$ is the largest challenge when analyzing MRI. Most existing breast segmentation methods are semi-automatic and have a limited ability to obtain accurate results. Automatic image segmentation is one of the most difficult processes in image processing. The reason is that the difficulties of removing bookmarks from noisy MRI and intensity inhomogeneity are a common problem within breast MRIs (21). Various techniques have been reported in the documentation of segmentation methods. The most commonly used method is the k-means clustering method (22). The performance of the methods relies on the contrast between the border regions $(21,23)$. It should be remembered that there is no universal segmentation method that can be applied to all images, and no segmentation method is perfect. In other words, as in image enhancement and restoration problems, the methods designed for image segmentation and the performance of these methods are varies depending on the appearance and application. For the first time in the breast MR literature, we used the EM method to segment the ROI, which is the crucial stage in CAD. EM is a popular iterative improvement algorithm for segmentation and model-based auditing method. Recently, studies showed that EM provides a good balance of image quality between low - and high-frequency features and determining the structure of the model and the parameters (24). The iteration alternates between performing an expectation (E) and maximization (M) for each parameter (25). The EM algorithm has previously been used in various modalities for breast cancer but was not used in breast MRI images. With EM, instead of dividing pixels into classes, it is possible to determine the number of components and estimate the mixture. Therefore, we used the EM algorithm to obtain a better image quality in the segmentation phase. In literature, the EM algorithm is known as one of the methods that can be calculated easily and efficiently (26). EM, which is used to cluster data sets that contain categorical and numerical features, was used considering that it would increase the estimated rate on MRI images including benign and malignant mass features. Because compared to other iterative techniques,
EM's statistical point of view modeling ability and to obtain the maximum likelihood estimation are stronger (27). In this way, this algorithm revealed the difference between benign and malignant mass features more clearly than we expected.

The feature extraction process is a dimension reduction process. The complexity of data is reduced to simpler data. Accurate feature extraction and appropriate system design are the factors that influence the success and performance of the result (28). Many feature selection techniques are frequently used now. Like the segmentation methods and classifiers, it is impossible to say which is the best algorithm for feature selection or extraction. It all depends on your application at hand (29). DT are algorithms that commonly used for classification and prediction. Classification is the most essential technique in data mining and widely used in various fields (30). Classification of data using the DT technique is a two-step process, which is training and testing. Training step, previously known training data is analyzed by the classification algorithm in order to create a model. In the testing step, test data are used to determine the accuracy of the classification rules or DT. If the accuracy is acceptable, the rules are used to classify new data (30). The most commonly used methods are KNN and SVM as classifiers (31). In addition to these, Yassin et al. (12), in their detailed review of the breast cancer literature, indicate that two studies use LDA and NB, while no studies use DT as their classification algorithm. C4.5 Algorithm is one of the most well-known DT algorithms. The purpose of these calculations is to determine the predictor class that provides the highest information. C4.5 DT classification was used as a CAD approach in our study and achieved a success rate of $95.24 \%$ in the differentiation of breast lesions. These three classification methods we used were similar in malignant lesions, but C4.5 was better in discrimination of the benign lesions. The size of the benign lesion was smaller than the malignant lesions and the contrast enhancement patterns were more variable. Therefore segmentation was more difficult in benign lesions. This result showed that the success rate of C4.5 could be increased with the use of EM. However, due to the small number of cases in the test group, statistically significant results could not be obtained.

\section{Study Limitations}

We had a small sample size, was not a large independent data set. Image analysis was performed from 2D images. However, some studies have shown that the results are similar in 2D and 3D image analysis. The manual placement of the ROI was also a limitation. Recently, automated software is available for ROI placement (32). Also, the large tumor size of the patients facilitated the detection and recognition of the tumor. Finally, Although a 
large number of data statistics and image post-processing restrictions, we achieved satisfactory results. We need to expand our findings in future studies.

\section{Conclusion}

Detecting and characterizing breast cancer can be assisted by using some computerized feature extraction and classification algorithms. Malignant tumors can be distinguished from a benign tumor on MRI with 95.24\% accuracy using EM segmentation and C4.5 classification. The number of unnecessary biopsies might be reduced with integration into the clinical workflow.

\section{Authorship Contributions}

Concept: T.I., Design: T.I., Data Collection or Processing: T.I., A.H.Y., Analysis or Interpretation: A.H.Y., H.E., S.K., Literature Search: T.I., A.H.Y., Writing: T.I.,

Conflict of Interest: The authors have no conflicts of interest to declare.

Financial Disclosure: The authors declared that this study has received no financial support.

\section{References}

1. Özmen V. Breast cancer in the world and Turkey. J Breast Health 2008;4:6-12

2. Berg WA. Benefits of screening mammography. JAMA 2010;303:168-9.

3. Sickles EA. Screening for breast cancer with mammography. Clin Imaging 1991;15:253-60.

4. Kuhl C. The current status of breast MR imaging. Part I. Choice of technique, image interpretation, diagnostic accuracy, and transfer to clinical practice. Radiology 2007;244:356-78.

5. Warner E, Hill K, Causer $P$, et al. Prospective study of breast cancer incidence in women with a BRCA1 or BRCA2 mutation under surveillance with and without magnetic resonance imaging. J Clin Oncol 2011;29:1664-9.

6. Kuhl CK, Schrading S, Strobel K, Schild HH, Hilgers RD, Bieling HB. Abbreviated breast magnetic resonance imaging (MRI): first postcontrast subtracted images and maximum-intensity projection-a novel approach to breast cancer screening with MRI. J Clin Oncol 2014;32:2304-10.

7. Wang S, Summers RM. Machine learning and radiology. Med Image Anal 2012;16:933-51.

8. Deurloo EE, Muller SH, Peterse JL, Besnard AP, Gilhuijs KG. Clinically and mammographically occult breast lesions on MR images: potential effect of computerized assessment on clinical reading. Radiology 2005;234:693-701.

9. Hadjiiski L, Sahiner B, Chan HP. Advances in computeraided diagnosis for breast cancer. Curr Opin Obstet Gynecol 2006;18:64-70.

10. Zaitoun NM, Aqel MJ. Survey on Image Segmentation Techniques. Procedia Computer Science 2015;65:797-806.
11. Doran SJ, Hipwell JH, Denholm R, et al. Breast MRI segmentation for density estimation: Do different methods give the same results and how much do differences matter? Med Phys 2017;44:4573-92.

12. Yassin NIR, Omran S, El Houby EMF, Allam H. Machine learning techniques for breast cancer computer aided diagnosis using different image modalities: A systematic review. Comput Methods Programs Biomed 2018;156:25-45.

13. Dempster AP, Laird NM, Rubin DB. Maximum Likelihood from Incomplete Data via the EM Algorithm. Series B (methodological) Journal of the Royal Statistical Society 1977;39:1-38.

14. Giger ML. Machine Learning in Medical Imaging. J Am Coll Radiol 2018;15:512-20.

15. Gillies RJ, Kinahan PE, Hricak H. Radiomics: Images Are More than Pictures, They Are Data. Radiology 2016;278:563-77.

16. Wong SH, Al-Hasani H, Alam Z, Alam A. Artificial intelligence in radiology: how will we be affected? Eur Radiol 2019;29:1413.

17. Crivelli P, Ledda RE, Parascandolo N, Fara A, Soro D, Conti M. A New Challenge for Radiologists: Radiomics in Breast Cancer. Biomed Res Int 2018;2018:6120703.

18. Chan HP, Sahiner $B$, Helvie MA, et al. Improvement of radiologists' characterization of mammographic masses by using computer-aided diagnosis: an ROC study. Radiology. 1999;212:817-27.

19. Aljabar P, Wolz R, Rueckert D. Manifold Learning for Medical Image Registration, Segmentation, and Classification. Machine learning in computer-aided diagnosis: Medical Imaging Intelligence and Analysis, IGI Global 2012. pp. 35172.

20. Middlebrooks EH, Tuna IS, Almeida L, et al. Structural connectivity-based segmentation of the thalamus and prediction of tremor improvement following thalamic deep brain stimulation of the ventral intermediate nucleus. Neuroimage Clin 2018;20:1266-73.

21. Rampun A, Scotney BW, Morrow PJ, Wang H, Winder J. Segmentation of breast MR images using a generalised 2D mathematical model with inflation and deflation forces of active contours. Artif Intell Med 2019;97:44-60

22. Kanungo T, Mount DM, Netanyahu NS, Piatko CD, Silverman R, Wu AY. An efficient k-means clustering algorithm: Analysis and implementation. IEEE Transactions on Pattern Analysis \& Machine Intelligence 2002;24:881-92.

23. Chen W, Giger ML, Bick U. A fuzzy c-means (FCM)-based approach for computerized segmentation of breast lesions in dynamic contrast-enhanced MR images. Acad Radiol 2006; 13:63-72

24. Gomi T, Koibuchi Y. Use of a Total Variation Minimization Iterative Reconstruction Algorithm to Evaluate Reduced Projections during Digital Breast Tomosynthesis. Biomed Res Int 2018;2018:5239082. 
25. Saha M, Chakraborty C, Arun I, Ahmed R, Chatterjee S. An Advanced Deep Learning Approach for Ki-67 Stained Hotspot Detection and Proliferation Rate Scoring for Prognostic Evaluation of Breast Cancer. Sci Rep 2017;7:3213.

26. Zhou J, Zhang J, Lu W. An Expectation Maximization algorithm for fitting the generalized odds-rate model to interval censored data. Stat Med 2017;36:1157-71.

27. Li H, Pan D, Chen CL. Reliability modeling and life estimation using an expectation maximization based wiener degradation model for momentum wheels. IEEE Trans Cybern 2015;45:955-63.

28. Deniz E, Şengür A, Kadiroğlu Z, Guo Y, Bajaj V, Budak Ü. Transfer learning based histopathologic image classification for breast cancer detection. Health Inf Sci Syst 2018;6:18.
29. Saha A, Yu X, Sahoo D, Mazurowski MA. Effects of MRI scanner parameters on breast cancer radiomics. Expert Syst Appl 2017;87:384-91.

30. Kesavaraj G, Sukumaran S. A study on classification techniques in data mining. 2013 Fourth International Conference on Computing, Communications and Networking Technologies (ICCCNT) 2013. pp. 1-7.

31. Nattkemper TW, Arnrich B, Lichte O, et al. Evaluation of radiological features for breast tumour classification in clinical screening with machine learning methods. Artif Intell Med 2005;34:129-39.

32. Whitney HM, Taylor NS, Drukker K, et al. Additive Benefit of Radiomics Over Size Alone in the Distinction Between Benign Lesions and Luminal A Cancers on a Large Clinical Breast MRI Dataset. Acad Radiol 2019;26:202-9. 